\title{
Identification of novel therapeutic targets in the secretome of ionizing radiation-induced senescent tumor cells
}

\author{
HYUN JUNG HWANG ${ }^{1 *}$, SEUNG HEE JUNG ${ }^{1 *}$, HYUNG CHUL LEE $^{1 *}$, NA KYUNG HAN ${ }^{2}$, \\ IN HWA BAE ${ }^{2}$, MINYOUNG LEE ${ }^{2}$, YOUNG-HOON HAN ${ }^{2}$, YOUNG-SUN KANG ${ }^{3,4}$, \\ SU-JAE LEE ${ }^{5}$, HEON JOO PARK ${ }^{6}$, YOUNG-GYU KO ${ }^{7}$ and JAE-SEON LEE ${ }^{1}$ \\ ${ }^{1}$ Department of Molecular Medicine and The Medical Research Center, Inha University College of Medicine, Incheon 22212; \\ ${ }^{2}$ Research Institute of Radiological and Medical Sciences, Korea Institute of Radiological and Medical Sciences, \\ Seoul 139-706; Departments of ${ }^{3}$ Biomedical Science and Technology and ${ }^{4}$ Veterinary Pharmacology and Toxicology, \\ College of Veterinary Medicine, Konkuk University, Seoul 143-701; ${ }^{5}$ Department of Life Science, \\ Research Institute for Natural Sciences, Hanyang University, Seoul 133-791; ${ }^{6}$ Department of Microbiology and

 \\ and Biotechnology, Korea University, Seoul 136-701, Republic of Korea
}

Received September 17, 2015; Accepted October 26, 2015

DOI: $10.3892 /$ or.2015.4473

\begin{abstract}
Cellular senescence is a state of irreversible growth arrest that can be triggered by multiple mechanisms, including telomere shortening, the epigenetic derepression of the INK4 $\alpha /$ ARF locus and DNA damage. Senescence has been considered a tumor-suppressing mechanism that permanently arrests cells at risk for malignant transformation. However, accumulating evidence shows that senescent cells have deleterious effects on the tissue microenvironment. Some of these effects could be attributed to the senescence-associated secretory phenotype that has the ability to promote tumor progression. However, secreted proteins from senescent tumor cells and their effects on the tumor microenvironment due to ionizing radiation (IR) exposure have not yet been fully elucidated. In the present study, we analyzed cytokines secreted from IR-induced senescent MCF7 cells by using cytokine microarrays and confirmed by western blot analysis that increased secretion of osteoprotegerin (OPG), midkine (MDK) and apolipoprotein E3 (ApoE3) occurs in these cells. Invasive, migratory and wound-healing activities were observed in MDA-MB-231 and MCF-10A cells following treatment with recombinant human OPG, MDK and ApoE3 proteins. Additionally, tube-formation activity was assessed in OPG-, MDK- and ApoE3-treated human umbilical vein
\end{abstract}

Correspondence to: Professor Jae-Seon Lee, Department of Molecular Medicine and The Medical Research Center, Inha University College of Medicine, Incheon 22212, Republic of Korea E-mail: jaeslee@inha.ac.kr

*Contributed equally

Keywords: cytokine, radiation, senescent cancer cells, TNFRSR11B, invasion, migration, wound healing, angiogenesis endothelial cells (HUVECs). We found that OPG, MDK and ApoE3 affected cell motility and tube-formation activity. Since OPG markedly affected cell motility, we examined the effect of senescent conditioned media containing neutralizing OPG antibodies on migration and wound-healing activity. Our results demonstrated that IR-induced senescent tumor cells influence the tumor microenvironment by increasing the production of cytokines, such as OPG, MDK and ApoE3. Furthermore, these data suggest that OPG is likely a promising target capable of reducing the deleterious effects on the tumor microenvironment during radiation therapy.

\section{Introduction}

Cellular senescence is irreversible cell cycle arrest, a process that was originally identified in normal human fibroblasts (1). It has been recently shown that various stresses, such as DNA damage, lack of nutrients, oxidative stress, improper cell contacts and oncogene activation, could trigger cellular senescence in normal and cancer cells (2). Thus, cellular senescence has been accepted as a general biological program in the last decade.

Senescent cells are metabolically active and exhibit characteristic flattened, enlarged morphologies with senescence-associated $\beta$-galactosidase activity. Senescent cells also undergo many molecular changes in gene expression, protein processing and chromatin organization $(3,4)$. Since a variety of DNA-damaging agents, including ionizing radiation (IR) and chemotherapeutic drugs, can prematurely induce cancer cell senescence, premature senescence might serve as a tumor-suppressing mechanism as potent as apoptosis both in vitro and in vivo (5). Senescent cells are detected in early-stage premalignant lesions in the tissues of mouse tumor models and human cancer patients, supporting the notion that cellular senescence is an anticancer barrier in early human tumorigenesis (6-8). 
As described above, mounting evidence supports that cellular senescence is a strong tumor-suppressing mechanism. However, senescent cancer cells have multiple facets facilitated by their altered secretory profiles. Collectively, these changes are referred to as the senescence-associated secretory phenotype (SASP) (9). The SASP is comprised of three major classes of secretory proteins, including chemokines and cytokines, matrix-remodeling proteases and growth factors (9). Senescent tumor cells promote cancer progression, stimulate angiogenesis, induce epithelial-to-mesenchymal transitions, facilitate tissue repair and contribute to cellular senescence $(10,11)$. The SASP could also trigger specific innate immune responses, which ultimately contribute to the elimination of senescent cancer cells $(12,13)$. Thus, the SASP could have both beneficial and detrimental effects on the tumor microenvironment.

Radiotherapy is one of the major regimens used to treat cancer patients. We previously reported that premature senescence is efficiently induced by IR treatment in a variety of carcinoma cell lines and in a human tumor xenograft mouse model (14-16). To minimize the side effects of radiotherapy, we must expand our knowledge of the SASP brought on by IR-induced senescent tumor cells. In the present study, we listed the top 20 cytokines that were increased in IR-induced senescent MCF7 cells using cytokine microarray analysis. From this list, we demonstrated that osteoprotegerin (OPG), midkine (MDK) and apolipoprotein E3 (ApoE3) can influence the tumor microenvironment and that OPG is likely a promising target capable of reducing the side effects associated with radiation therapy.

\section{Materials and methods}

Reagents and antibodies. Recombinanthuman proteins of OPG, MDK and ApoE3 were purchased from Biovision (Milpitas, CA, USA). Phospho-pRb antibody was purchased from Cell Signaling Technology (Danvers, MA, USA). p53 antibody was purchased from Leica Biosystems (Wetzlar, Germany). p21 antibody was purchased from Santa Cruz Biotechnology (CA, USA). Actin antibody was purchased from ABM (Richmond, BC, Canada). The neutralizing OPG antibody was purchased from R\&D Systems (Minneapolis, MN, USA). The Matrigel matrix and Transwell upper chambers were purchased from Corning (Cambridge, MA, USA). The Ultracel-3K filter was purchased from Millipore (Billerica, MA, USA). Crystal violet dye was purchased from Sigma-Aldrich (St. Louis, MO, USA).

Cell culture and irradiation. MCF-7 cells were cultured in Dulbecco's modified Eagle's medium (DMEM) (Welgene, Inc., Daegu, Korea), MDA-MB-231 cells were cultured in RPMI-1640 media (Welgene, Inc.), and human umbilical vein endothelial cells (HUVECs) were grown in EBM-2 media (Lonza, Walkersville, MD, USA) supplemented with $10 \%$ fetal bovine serum (FBS) and 1\% penicillin and streptomycin (all from Welgene, Inc.) at $37^{\circ} \mathrm{C}$ in a $5 \% \mathrm{CO}_{2}$ incubator. For irradiation, cells were exposed to $\gamma$-rays with a ${ }^{137} \mathrm{Cs} \gamma$-ray source (Modle 68; JL Shepherd and Associates, Glenwood, CA, USA) at a dose rate of 200-300 cGy/min.

Senescence-associated $\beta$-galactosidase staining. Cells were washed in phosphate-buffered saline (PBS), fixed at room temperature (RT) for 3-5 min in 3.7\% formaldehyde, washed with $\mathrm{PBS}$, and incubated at $37^{\circ} \mathrm{C}$ in $0 \% \mathrm{CO}_{2}$ with fresh staining solution consisting of $1 \mathrm{mg} / \mathrm{ml}$ 5-bromo-4-chloro-3-indolyl $\beta$-D-galactoside (X-Gal, from a stock solution of $20 \mathrm{mg} / \mathrm{ml}$ in dimethylformamide) in $40 \mathrm{mM}$ citric acid/sodium phosphate, pH $6.0,5 \mathrm{mM}$ potassium ferrocyanide, $5 \mathrm{mM}$ potassium ferricyanide, $150 \mathrm{mM} \mathrm{NaCl}$ and $2 \mathrm{mM} \mathrm{MgCl}{ }_{2}$. Staining was maximal at $12-16 \mathrm{~h}$.

Collection of conditioned media (CM). MCF7 cells were seeded at $5 \times 10^{4}$ cells $/ \mathrm{ml}$ in $100 \mathrm{~mm}$ culture dishes prior to overnight culture and exposed to either 0 or 6 Gy radiation. Cells were kept in a $\mathrm{CO}_{2}$ incubator for 3 days, washed with PBS three times and incubated in $5 \mathrm{ml}$ of fresh serum-free media without antibiotics. CM were collected after an additional $24 \mathrm{~h}$ of incubation, centrifuged to remove any residual cells, and filtered with a syringe filter with a pore size of $0.2 \mu \mathrm{m}$. Filtered CM were concentrated 5- or 10-fold using a Centricon-10 concentrator (Millipore) at $4^{\circ} \mathrm{C}$. After collecting $\mathrm{CM}$, the number of cells on the dish was counted and normalized to the volume of $\mathrm{CM}$ used in each experiment.

Sample preparation for cytokine microarray analysis. Proteins from CM were obtained using a gel matrix column that was included in the antibody array assay kit (Full Moon Biosystems, Sunnyvale, CA, USA). The column was vortexed for $5 \mathrm{sec}$ and hydration-treated for $60 \mathrm{~min}$ at RT. After hydration, the column was centrifuged at $750 \mathrm{x}$ g for $2 \mathrm{~min}$. After centrifugation, the column was placed into a collection tube, and $100 \mu \mathrm{l}$ of the protein sample was transferred into the column and centrifuged at $750 \mathrm{xg}$ for $2 \mathrm{~min}$. The concentration of the purified sample was measured with the bicinchoninic acid assay (BCA) protein assay kit (Pierce, Rockford, IL, USA) using a NanoPhotometer ${ }^{\mathrm{TM}}$ (Implen, UK), and the purity of the purified sample was confirmed with an ultraviolet spectrophotometer.

Cytokine microarray analysis. For each sample, $75 \mu 1$ of labeling buffer was added to a 50- $\mu \mathrm{g}$ sample of protein. Subsequently, the sample was treated with $3 \mu \mathrm{l}$ of $10 \mu \mathrm{g} / \mu \mathrm{l}$ biotin/DMF solution and incubated at RT for $1 \mathrm{~h}$ with mixing. To stop the reaction, the sample was treated with $35 \mu 1$ of stop reagent and incubated at RT for 30 min with mixing. The cytokine-profiling antibody array slide (Full Moon Biosystems) was treated with $30 \mathrm{ml}$ of blocking solution in a Petri dish and incubated at $55 \mathrm{rpm}$ for $1 \mathrm{~h}$ at RT with shaking. After blocking, the slide was rinsed with Milli-Q grade water. The labeled sample was mixed with $6 \mathrm{ml}$ of coupling solution. The blocked array slide was incubated in coupling mixture at $60 \mathrm{rpm}$ for $2 \mathrm{~h}$ at RT with shaking in a coupling dish. After coupling, the slide was washed six times with $30 \mathrm{ml}$ of washing solution in a Petri dish on a shaker at $55 \mathrm{rpm}$ for 5 min and rinsed with Milli-Q grade water. Thirty microliters of $0.5 \mathrm{mg} / \mathrm{ml} \mathrm{Cy} 3$-streptavidin (GE Healthcare, Chalfont St. Giles, UK) was mixed with $30 \mathrm{ml}$ of detection buffer. The coupled array slide was incubated with detection mixture in a Petri dish at $55 \mathrm{rpm}$ for $20 \mathrm{~min}$ at RT with shaking, washed six times with $30 \mathrm{ml}$ of washing solution in a Petri dish at $55 \mathrm{rpm}$ for $5 \mathrm{~min}$ with shaking and rinsed with Milli-Q grade water. 
Table I. List of the top 20 cytokines with increased levels of secretion following IR-induced senescence in MCF7 cells.

\begin{tabular}{lcc}
\hline & $\begin{array}{c}\text { Fold-change } \\
\text { (senescent cells/ } \\
\text { List of cytokines }\end{array}$ & Gene ID \\
\hline Serpin peptidase inhibitor clade E member 1 (Serpine1) & 5.15 & P05121 \\
Midkine (MDK) & 4.56 & P21741 \\
Apolipoprotein E3 (ApoE3) & 3.36 & Q13791 \\
Transforming growth factor $\beta 1$ (TGF- $\beta$ 1) & 3.16 & P01137 \\
Galectin-3 & 2.99 & P17931 \\
Galectin-1 & 2.61 & P09382 \\
Osteoprotegerin (OPG) & 2.55 & O00300 \\
Receptor activator of nuclear factor kB ligand (RANKL) & 2.49 & O14788 \\
Ferritin heavy polypeptide 1 (FTH1) & 2.14 & P02794 \\
Nicotinamide phosphoribosyltransferase (NAMPT) & 1.98 & P43490 \\
Insulin-like growth factor binding protein 5 (IGFBP5) & 1.94 & P24593 \\
Aminoacyl tRNA synthetase complex-interacting multifunctional protein 1 (AIMP1) & 1.88 & Q12904 \\
Chemokine (C-X3-C motif) ligand 1 (CX3CL1) & 1.83 & P78423 \\
Fibroblast growth factor 5 (FGF5) & 1.67 & P12034 \\
Kallikrein-related peptidase 3 (KLK3) & 1.57 & P07288 \\
Nephroblastoma overexpressed (NOV) & 1.51 & P48745 \\
Thyroglobulin (TG) & 1.49 & P01266 \\
Adenomatous polyposis coli (APC) & 1.48 & P25054 \\
Betacellulin (BTC) & 1.47 & Q86UF5 \\
Myostatin (MSTN) & 1.46 & O14793 \\
\hline
\end{tabular}

Cytokine microarray data acquisition and analysis. We scanned the slide using a GenePix 4000B scanner (Molecular Devices, Sunnyvale, CA, USA). The slides were completely dried and scanned within $24-48 \mathrm{~h}$ after drying. The slides were scanned at $10-\mu \mathrm{m}$ resolution, with optimal laser power and PMT. Using GenePix Software (Molecular Devices), grids were applied to the scanned images, and the pixels were quantified. The obtained numerical data were analyzed using Genowiz 4.0 ${ }^{\mathrm{TM}}$ (Ocimum Biosolutions, Indianapolis, IN, USA). Following analysis, the protein data were annotated using UniProt DB (www.uniprot.org).

Western blot analysis. To prepare samples for western blot analysis, CM was collected and boiled with sodium dodecyl sulfate (SDS) sample buffer [250 mM Tris-HCl, 8\% (w/v) SDS, $40 \%$ (v/v) glycerol, $8 \%$ (v/v) $\beta$-mercapto-ethanol, and $0.02 \%$ (w/v) bromophenol blue] and subjected to SDS-polyacryamide gel electrophoresis. Thereafter, the gel was transferred to a nitrocellulose membrane for immunoblotting. Membranes were blocked with $5 \%(\mathrm{w} / \mathrm{v})$ skim milk in Tris-buffered saline with $0.1 \%(\mathrm{v} / \mathrm{v})$ Tween-20 (TBST) solution and incubated with the appropriate primary and secondary antibodies for $1 \mathrm{~h}$ at RT. After washing, the membranes were subjected to enhanced chemiluminescence assays (Thermo Fisher Scientific, Waltham, MA, USA) and exposed to X-ray film (Agfa Gevaert NV, Mortsel, Antwerp, Belgium) to illuminate the protein bands.

In vitro cell migration and invasion assays. The Transwell migration and Matrigel invasion assays were conducted using the methods described by the manufacturers using modified Boyden chambers with 8- $\mu \mathrm{m}$ pore filter inserts for 24-well plates (Costar, Cambridge, MA, USA). For the invasion assay, filters were pre-coated with $10 \mu \mathrm{l}$ ice cold $0.1 \%$ Matrigel (BD Biosciences, San Jose, CA, USA) in DPBS. Cells $\left(1 \times 10^{5}\right)$ in $200 \mu \mathrm{l}$ of serum-free media were added to the upper chamber. For the negative and positive control groups, cells were incubated in serum-free media and media containing $1 \%$ serum, respectively. For the experimental groups, the lower chamber was filled with $600 \mu \mathrm{l}$ of either serum-free media containing $1 \mu \mathrm{g} / \mathrm{ml}$ of protein (OPG, MDK, or ApoE3) or CM containing $20 \mu \mathrm{g} / \mathrm{ml}$ of neutralizing OPG antibody. After $16 \mathrm{~h}$ of incubation, the membranes were incubated with $3.7 \%$ formaldehyde solution for $20 \mathrm{~min}$ to fix the cells and stained with crystal violet solution for $30 \mathrm{~min}$. Subsequently the non-invasive cells in the inserts were removed with cotton swabs. The invasive cells on the underside of the membrane were counted using a light microscope (Olympus CKX41; Olympus, Shinjuku, Tokyo, Japan) and photographed.

Wound-healing assay. MDA-MB-231 cells were dispensed into 12-well plates at a high density and incubated in complete media in a $\mathrm{CO}_{2}$ incubator for $16 \mathrm{~h}$. Then, the media were replaced with serum-free media for an additional $16 \mathrm{~h}$. After scraping the cell monolayer with a sterile $200-\mu 1$ pipette tip, the debris was removed by washing with DPBS, and the wells were refilled with $1 \mathrm{ml}$ of fresh serum-free media. For the negative and positive control groups, cells were incubated in serum-free media and media containing $10 \%$ serum for $30 \mathrm{~h}$, 
A



C


D


B
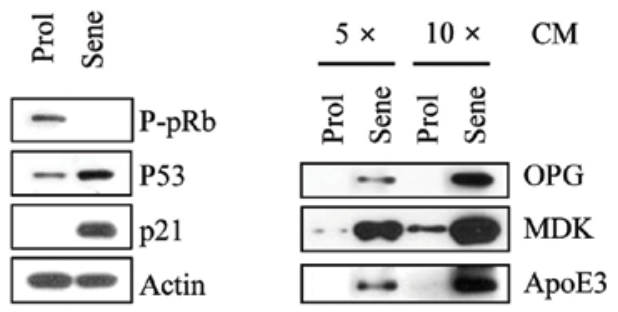

$\mathbf{E}$
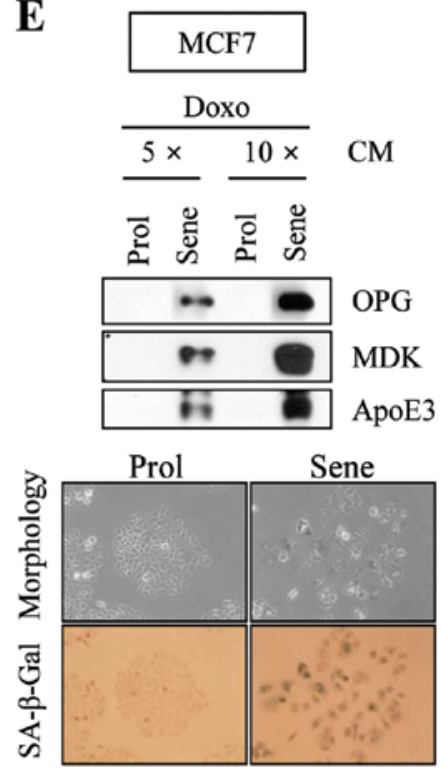

Figure 1. Western blot analysis of differentially secreted proteins in proliferating and IR-induced senescent MCF7, HCT116, and H460 cells. (A) Cell morphology, senescence-associated $\beta$-galactosidase (SA- $\beta$-Gal) staining, and western blot analysis in IR-induced senescent MCF7 cells. Representative images are shown. (B) Western blot analysis of conditioned media (CM) collected from MCF7 cells during proliferation (Prol) and IR-induced senescence (Sene). CM was concentrated 5- and 10-fold prior to western blot analysis. (C and D) Western blot analyses of senescent CM obtained from IR-induced senescence in HCT116 (C) and H460 cells (D). Specific antibodies were applied as indicated to detect each protein in western blot analyses. (E) Western blot analysis of CM collected from MCF7 cells during proliferation and doxorubicin-induced senescence (upper) and cell morphology and SA- $\beta$-Gal activity (lower) in doxorubicin-induced senescent MCF7 cells.

respectively. For the experimental groups, cells were incubated in either serum-free media containing $1 \mu \mathrm{g} / \mathrm{ml}$ of recombinant protein (OPG, MDK, or ApoE3) or CM containing $20 \mu \mathrm{g} / \mathrm{ml}$ of neutralizing OPG antibody for $30 \mathrm{~h}$. Cells were washed with DPBS, fixed with $3.7 \%$ paraformaldehyde for $30 \mathrm{~min}$, and stained with $0.5 \%$ (w/v) crystal violet (Sigma-Aldrich). After washing with water, cells were air dried and inspected with a light microscope (Olympus CKX41; Olympus). Data are expressed as percentage of wound healing ( $\mathrm{WH} \%$ ) calculated by dividing the migrated distance by the scratched distance.

Tube formation assay. Each well of a 48-well plate was coated with $100 \mu$ l of Matrigel (BD Biosciences) and incubated at $37^{\circ} \mathrm{C}$ for $30 \mathrm{~min}$. For the negative and positive control groups, gels were overlaid with HUVECs $\left(4 \times 10^{4}\right)$ suspended in serum-free media and media containing $0.5 \%$ serum and growth factors (VEGF, EGF, FGF and IGF), respectively. For the experimental groups, gels were overlaid with HUVECs $\left(4 \times 10^{4}\right)$ suspended in media containing $0.5 \%$ serum, growth factors (VEGF, EGF, FGF and IGF), and $1 \mu \mathrm{g} / \mathrm{ml}$ of recombinant protein (OPG,
MDK, or ApoE3). After $16 \mathrm{~h}$ of incubation, the area of tube growth was photographed under a microscope. To quantify HUVEC tube formation, the numbers of branch points and branches were counted, and the tube area was quantified using Fuji Multi Gauge V2.3 software (Fuji, Tokyo, Japan).

Statistical analysis. Data are presented as mean \pm SD from at least three separate experiments. Results were considered significant when $\mathrm{P} \leq 0.05$ was obtained. All the statistical analyses were performed using SPSS 19 (IBM ${ }^{\circledR}$ SPSS $^{\circledR}$ Statistics, IBM Corporation, Somers, NY, USA).

\section{Results}

Identification of cytokines from IR-induced senescent MCF7 cells. To examine cytokines differentially secreted from IR-induced senescent cancer cells, we exposed MCF7 breast carcinoma cells to 6 Gy of IR. IR-exposed MCF7 cells exhibited specific characteristics of cellular senescence, such as enlarged, flattened morphological changes and positive 
A
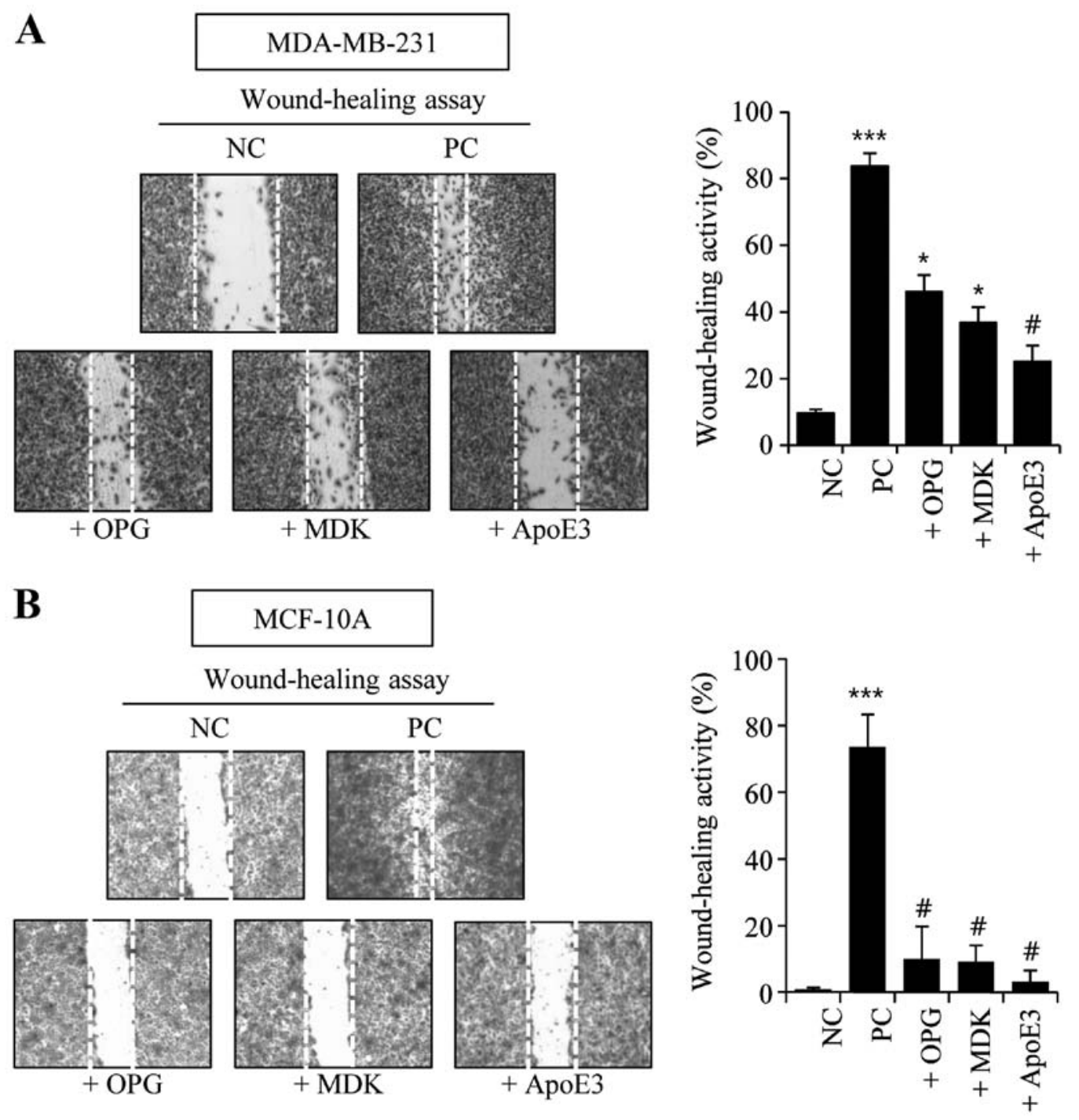

Figure 2. Effects of recombinant human OPG, MDK and ApoE3 proteins on wound-healing activity. The effects of recombinant OPG, MDK and ApoE3 on wound healing in MDA-MB-231 (A) and MCF7 cells (B). After treatment with each recombinant human protein as described in Materials and methods, wound-healing activity was analyzed. NC and PC indicate the negative and positive controls, respectively. Representative images are shown in each panel. Graphs show quantitative data from three independent experiments. Statistical significance is indicated by ${ }^{*} \mathrm{P}<0.05,{ }^{* * *} \mathrm{P}<0.01$ and ${ }^{* * * *} \mathrm{P}<0.001$ compared to the controls. OPG, osteoprotegerin; MDK, midkine; ApoE3, apolipoprotein E3.

senescence-associated $\beta$-galactosidase staining (Fig. 1A). These cells also showed pRb hypophosphorylation, p53 accumulation and p21 induction in western blot analyses (Fig. 1A). Together, these results indicate that 6 Gy of IR efficiently induced premature senescence in MCF7 cells.

To identity cytokines differentially secreted in proliferating and senescent MCF7 cells, we collected CM from proliferating and senescent cells and analyzed their respective cytokine profiles using a cytokine-profiling array. Table I shows the top 20 cytokines that were increased in IR-induced senescent MCF7 cells. From this list of cytokines, we selected MDK and ApoE3 for further analysis since they have not previously been identified as factors that demonstrate increased secretion in senescent tumor cells. We also selected OPG, which is reported to increase in secretion in senescent human fibroblasts and epithelial cells (17). To confirm that their secretion levels increased in IR-induced senescent MCF7 cells, we performed western blot analysis after normalizing the volume of $\mathrm{CM}$ with the number of cells on the dish following CM collection. The levels of MDK, ApoE3 and OPG were elevated in senescent CM compared to proliferating CM (Fig. 1B). When we examined the secretion of MDK, ApoE3 and OPG in other types of cancer cell lines, such as HCT116 colon carcinoma and H460 lung carcinoma cells, under IR-induced senescence conditions, we observed elevated levels of these proteins (Fig. 1C and D). Furthermore, we found that OPG, MDK and ApoE3 were secreted, under anticancer drug, doxorubicin-induced senescence condition (Fig. 1E).

Effects of $O P G, M D K$, and ApoE3 on migration, invasion and wound healing in MDA-MB-231 cells. To test the effects of secreted MDK, ApoE3 and OPG on the tumor microenvironment, we treated MDA-MB-231 breast carcinoma, MCF-10A breast epithelial and HUVEC endothelial cells with recombinant human MDK, ApoE3 and OPG proteins and observed phenotypes related to cell motility, invasiveness and angiogenesis. We first examined the effects of these proteins on wound-healing activity. For the negative 
A
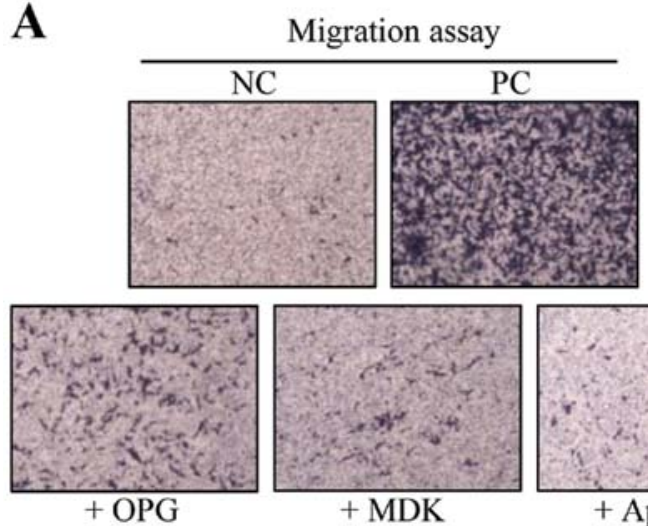

+ MDK



B

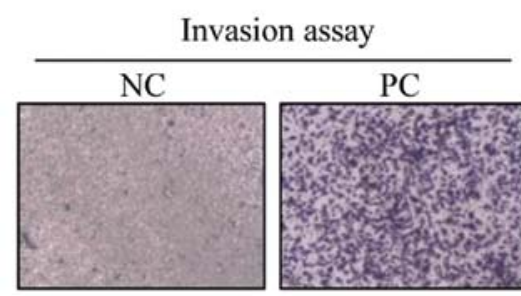

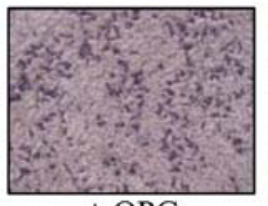

$+\mathrm{OPG}$



$+\mathrm{MDK}$



$+\mathrm{ApoE} 3$


Figure 3. Effects of recombinant human OPG, MDK and ApoE3 proteins on cell migration and invasion. MDA-MB-231 cells were treated with each recombinant human protein as described in Materials and methods, and cell migration (A) and invasion (B) assays were performed. NC and PC indicate the negative and positive controls, respectively. Representative images are shown in each panel. Graphs show quantitative data from three independent experiments. Statistical significance is indicated by ${ }^{*} \mathrm{P}<0.05,{ }^{* *} \mathrm{P}<0.01$ and ${ }^{* * * *} \mathrm{P}<0.001$ compared to the controls. OPG, osteoprotegerin; MDK, midkine; ApoE3, apolipoprotein E3.

and positive control groups, MDA-MB-231 and MCF7 cells were incubated in serum-free media and media containing $10 \%$ serum, respectively. Simultaneously, additional batches of cells were incubated in serum-free media containing $1 \mu \mathrm{g} / \mathrm{ml}$ of OPG, MDK, or ApoE3 recombinant proteins. OPG-, MDK- and ApoE3-treated groups clearly showed increased wound-healing activity in the MDA-MB-231 cells (Fig. 2A). However, the wound-healing activity in OPG-, MDK-, or ApoE3-treated MCF-10A cells was not significantly changed compared to the negative control cells (Fig. 2B).

Next, we conducted migration and invasion assays in OPG-, MDK- and ApoE3-treated MDA-MB-231 cells. Both the migratory and invasive activities were increased following treatment with OPG, MDK, or ApoE3 (Fig. 3). These activities were increased the most in OPG-treated cells, whereas ApoE3-treated cells had the least impact on migration and invasiveness (Fig. 3).

Effects of $O P G, M D K$ and ApoE3 on tube formation in HUVECs. To identify the possible effects of secreted OPG, MDK and ApoE3 on angiogenesis in the tumor microenvironment, we assayed tube formation. For the negative and positive control groups, gels were overlaid with HUVECs $\left(4 \times 10^{4}\right)$ suspended in serum-free media and media containing $0.5 \%$ serum and growth factors (VEGF, EGF, FGF and IGF), respectively. For the OPG-, MDK-, and ApoE3-treated groups, gels were overlaid with HUVECs $\left(4 \times 10^{4}\right)$ suspended in media containing $0.5 \%$ serum, growth factors (VEGF, EGF, FGF and IGF), and $1 \mu \mathrm{g} / \mathrm{ml}$ of OPG, MDK, or ApoE3 recombinant proteins. Tube-forming activity clearly increased following treatment with OPG, MDK and ApoE3 (Fig. 4A). The graphs in Fig. 4B show the quantifications of tube-forming activity in the OPG-, MDK- and ApoE3-treated HUVECs. OPG-treated cells exhibited the greatest increases in branch point number, branch number and tube area (Fig. 4). These data revealed that OPG, MDK and ApoE3 secreted from senescent cancer cells likely affect angiogenic activity in neighboring endothelial cells.

Effects of the neutralizing OPG antibody on migration and wound healing in MDA-MB-231 cells. Since OPG was the most effective at increasing wound healing, invasion and migration in MDA-MB-231 carcinoma cells as well as tube formation in HUVECs, we hypothesized that OPG is one of 
A

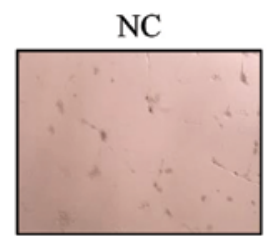

PC


+ MDK


B
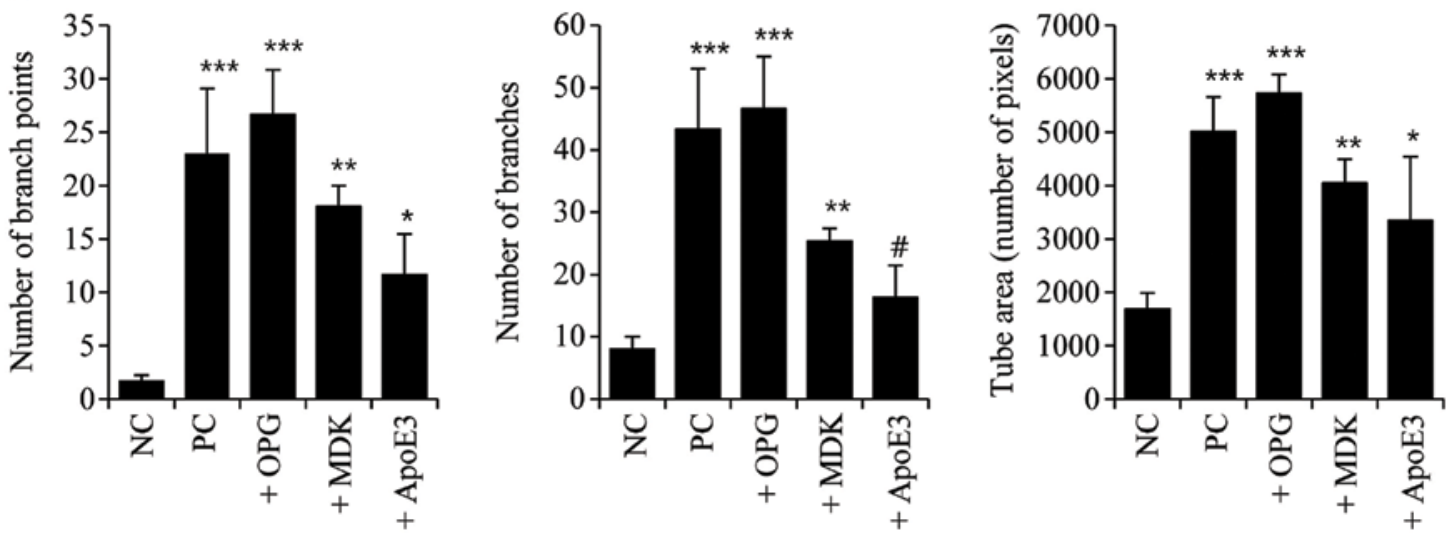

Figure 4. Effects of recombinant human OPG, MDK and ApoE3 proteins on tube-formation activity. (A) Tube-formation activity in HUVECs. Following treatments with each recombinant protein as described in Materials and methods, tube-formation activities were observed. Representative images are shown. (B) Quantifications of tube-forming activity. To quantify HUVEC tube formation, the numbers of branch points and branches were counted, and the area of the tubes were quantified using Fuji Multi Gauge V2.3 software. Data were obtained from three independent experiments and analyzed for statistical significance. NC and PC indicate the negative and positive controls, respectively. Representative images are shown. OPG, osteoprotegerin; MDK, midkine; ApoE3, apolipoprotein E3.

the most detrimental factors to the tumor microenvironment. Thus, we examined the role of OPG in senescence using CM and a neutralizing antibody against OPG (Neut OPG Ab). MDA-MB-231 cells were incubated in CM in both the absence and presence of $20 \mu \mathrm{g} / \mathrm{ml}$ Neut OPG Ab. For the negative and positive control groups, cells were incubated in serum-free media and media containing $1 \%$ serum, respectively. When we assessed the migratory activity of MDA-MB-231 cells in OPG-depleted CM, senescence-associated migratory activity was diminished (Fig. 5A). Additionally, the presence of the Neut OPG Ab also diminished senescence-associated wound-healing activity (Fig. 5B).

\section{Discussion}

The development of cancer is not dictated solely by cell growth. The tumor microenvironment is an indispensable participant in the neoplastic process, fostering cell proliferation and survival $(18,19)$. Normal cells can be recruited to the tumor microenvironment to aid malignant progression (20). Additionally, tumor cells produce various cytokines and chemokines that stimulate the innate immune response and other receptors necessary for invasion, migration and metastasis (18). Multiple mechanisms of intercellular communication in the tumor microenvironment can contribute both positive and negative signals to the tumor (20). Therefore, multiple therapeutic approaches to target different cell types in the tumor microenvironment are needed.

Cellular senescence, which is an extremely stable form of cell cycle arrest, could be triggered by exposures to various stresses, including telomere attrition, DNA damage, oxidative stress, lack of nutrition and improper cell contacts (21).
Given that neoplastic transformation involves events that inhibit senescence, tumor cells are believed to lose the ability to senesce. However, tumor cells can be triggered to undergo cellular senescence following treatments with anticancer drugs, radiation, DNA-damaging agents, or genetic manipulation (22). Currently, cellular senescence is recognized as a tumor-suppressing mechanism. Accumulating evidence has shown that cellular senescence is as potent as apoptosis at depriving the self-renewal capacity of cancer cells after radiotherapy and chemotherapy (23-25). Thus, triggering senescence is considered a key component of therapeutic interventions necessary for treating cancer, and potential senescence-inducing small molecules are currently in development (26).

Senescent cells undergo a variety of characteristic changes in cellular morphology, cell size, gene expression pattern, granularity, chromatin configuration and enzyme activity. Additionally, despite the importance of cellular senescence in cancer treatments, senescent cells paradoxically secrete factors that alter the tissue microenvironment and favor tumor growth (27-30). The SASP allows tumor cells to proliferate inappropriately, invade surrounding tissues, and reinforce senescence in cell-autonomous and -nonautonomous manners (10,27,31-33). The SASP also cell-nonautonomously affects angiogenesis and the infiltration of immune cells $(23,34,35)$. Since a variety of inflammatory cytokines comprise the SASP, there are at least four major processes by which cellular senescence is thought to contribute to tumor suppression, aging, tumor promotion and tissue repair $(10,11)$.

Currently, exposure to IR is being increased not only for cancer treatments, but also for diagnostic and occupational 
$\mathbf{A}$
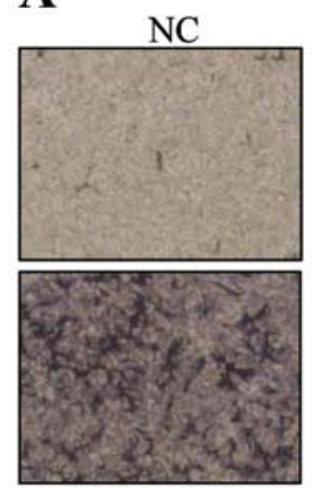

Sene CM
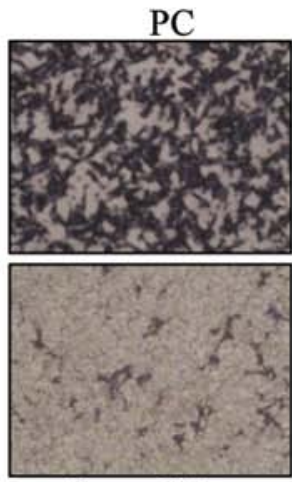

Sene CM

+ Neut OPG Ab

B




Sene $\mathrm{CM}$



Sene CM

+ Neut OPG Ab


Figure 5. Effects of the neutralizing osteoprotegerin (OPG) antibody on cell mobility and wound-healing activity in MDA-MB-231 cells. (A) The effect of the neutralizing OPG antibody on cell migration. Cells were treated with either 5-fold concentrated conditioned media (CM) or 5-fold concentrated CM containing $20 \mu \mathrm{g} / \mathrm{ml}$ of neutralizing OPG antibody (Neut OPG Ab). After $16 \mathrm{~h}$ of incubation, the number of migratory cells was quantified. Representative images are shown. The graph shows the quantifications of three independent experiments. (B) The effect of the neutralizing OPG antibody on wound-healing activity. Cells were treated with either CM or CM containing $20 \mu \mathrm{g} / \mathrm{ml}$ of neutralizing OPG antibody. Wound-healing activity was examined $16 \mathrm{~h}$ following treatment. CM was collected from IR-induced senescent MCF7 cells as described in Materials and methods. Data were obtained from three independent experiments and analyzed for statistical significance. NC and PC indicate the negative and positive controls, respectively. Representative images are shown.

reasons. Epithelial, mesenchymal, and tumor cells derived from theses tissues primarily respond to IR by initiating senescence $(14,36,37)$. A long-standing question regarding the bystander effects exhibited by unirradiated cells raises the possibility that the observed irradiation effects might be attributed to the SASP mediated by IR-induced senescent tumor cells $(37,38)$.

The major factors secreted by senescent cells are inflammatory cytokines, chemokines, growth factors, matrix metalloproteinases, and their inhibitors, such as IL-1, IL-6, IL-8, GRO, MCP-1, VEGF, MMP-1, MMP-3, MMP-10 and PAI $(34,36)$. However, secreted factors from IR-induced senescent cancer cells and their functions in the tumor microenvironment have not yet been fully elucidated. We previously identified novel proteins secreted from IR-induced senescent MCF7 cells using proteomic techniques and elucidated their roles in the tumor microenvironment (38). To extend our knowledge about the secretome of IR-induced senescent cancer cells in this study, we profiled the top 20 cytokines from IR-induced senescent cancer cells (Table I) and selected OPG, MDK and ApoE3 for further investigation by examining their effects on the tumor microenvironment during radiotherapy. Furthermore, we verified that the secretions of OPG, MDK, and ApoE3 are commonly increased in MCF7 
breast, H460 lung and HCT116 colon carcinoma cell lines following premature senescence induced by exposure to $6 \mathrm{~Gy}$ IR.

OPG, an essential secreted protein in bone, was originally identified for the role it plays as a decoy receptor for the receptor activator of nuclear factor- $\kappa \mathrm{B}$ ligand (RANKL) (39). The RANKL-RANK-OPG system is important for bone homeostasis, as it regulates osteoclasts (40). However, OPG acts in various biological functions due to its ability to bind to additional ligands in vascular, immune, and tumor tissues. OPG is also known to be involved in cell survival, cell proliferation and cell migration $(41,42)$. Specifically, OPG increases endothelial cell survival and migration and has metastasis-promoting effects $(17,42)$. However, the role of OPG in cancer remains unclear. There is conflicting evidence of indirect antitumoral effects and pro-tumoral effects mediated by TRAIL inhibition (43). Coppé et al (34) reported that OPG secretion increased following replicative senescence induced by 10 Gy of IR or various oncogenes; however, they did not validate the effects of secreted OPG on the tumor microenvironment. In the present study, we demonstrated that OPG exhibits pro-tumoral effects evidenced by the fact that treatment of CM with neutralizing OPG antibodies successfully diminished the pro-tumoral effects of senescence-inducing CM. Our results indicate that OPG secreted from IR-induced senescent cancer cells likely plays a crucial role in promoting the migration of neighboring cancer cells and that OPG could be a pivotal therapeutic target that can overcome the detrimental effects of senescent cancer cells generated by radiotherapy.

MDK, a heparin-binding growth factor, was originally identified in embryonal carcinoma cells. MDK expression is increased during digestion and decreased thereafter $(44,45)$. Whereas MDK expression is low in normal adult tissues, it is highly expressed in various cancers, indicating that MDK impacts tumor development $(44,46,47)$. Additionally, it has been demonstrated that MDK is involved in many biological processes, including development, inflammation, tissue protection, and blood pressure regulation (45).

ApoE has been identified as an essential constituent of plasma lipoproteins that is responsible for cholesterol transport and metabolism (48). ApoE is also involved in several biological processes not directly related to cholesterol, such as cell proliferation, the inflammatory response and endocytosis $(49,50)$. ApoE is produced mainly in the liver, adrenal glands, kidney, and macrophages, and secreted ApoE serves as an autocrine and paracrine growth factor (51). ApoE has four isoforms: ApoE1, E2, E3 and E4, which differ at amino acid residues 112 and 158 (52). The most prevalent and well-established genetic risk factor associated with ApoE is Alzheimer's disease. However, differences in the structure and function between the ApoE isoforms remains an open question.

To our knowledge, this reports provides the first evidence that there is increased secretion of MDK and ApoE3 from senescent tumor cells. We demonstrated that OPG, MDK and ApoE3 impact the tumorigenic, metastatic and angiogenic capabilities of the cells. Thus, we propose that OPG, MDK and ApoE3 could be promising therapeutic targets for modulating the pro-tumoral effects of secreted factors from senescent tumor cells during radiotherapy. Furthermore, when cancer cells senesce following treatments with the anticancer drug, doxorubicin, we found that OPG, MDK and ApoE3 were secreted. These results indicate that OPG, MDK and ApoE3 could be applied to senescent cancer cells as therapeutic agents that could diminish the side effects of radiotherapy and chemotherapy.

The relative concentrations of factors secreted by senescent tumor cells as well as the concentrations of other components in the tumor microenvironment likely determine whether the overall effects of a senescence-inducing secretome is pro-tumoral or antitumoral. Despite recent advances in our knowledge of the senescence secretome, many questions still remain. To minimize the detrimental effects of therapy-induced senescence or prosenescence therapy, we must validate the most pro-tumoral secreted factors and develop therapeutics that take advantage of these validated targets in vitro and in vivo.

\section{Acknowledgements}

The present study was supported by the Nuclear Research and Development Program (grant no. 2012M2B2B1055637) and the Medical Research Center (MRC) (grant no. 2014009392) of the National Research Foundation of Korea (NRF) funded by the Korean government (MSIP) and the Inha University Research Grant (grant no. INHA-51377).

\section{References}

1. Ben-Porath I and Weinberg RA: The signals and pathways activating cellular senescence. Int J Biochem Cell Biol 37: 961-976, 2005.

2. Liu S, Liu S, Wang X, Zhou J, Cao Y, Wang F and Duan E: The PI3K-Akt pathway inhibits senescence and promotes self-renewal of human skin-derived precursors in vitro. Aging Cell 10: 661-674, 2011.

3. Salama R, Sadaie M, Hoare M and Narita M: Cellular senescence and its effector programs. Genes Dev 28: 99-114, 2014.

4. Campisi J and d'Adda di Fagagna F: Cellular senescence: When bad things happen to good cells. Nat Rev Mol Cell Biol 8: 729-740, 2007.

5. Deng Y, Chan SS and Chang S: Telomere dysfunction and tumour suppression: The senescence connection. Nat Rev Cancer 8: 450-458, 2008.

6. Michaloglou C, Vredeveld LC, Soengas MS, Denoyelle C, Kuilman T, van der Horst CM, Majoor DM, Shay JW, Mooi WJ and Peeper DS: BRAFE600-associated senescence-like cell cycle arrest of human naevi. Nature 436: 720-724, 2005.

7. Braig M, Lee S, Loddenkemper C, Rudolph C, Peters AH, Schlegelberger B, Stein H, Dörken B, Jenuwein T and Schmitt CA: Oncogene-induced senescence as an initial barrier in lymphoma development. Nature 436: 660-665, 2005.

8. Sun P, Yoshizuka N, New L, Moser BA, Li Y, Liao R, Xie C, Chen J, Deng Q, Yamout M, et al: PRAK is essential for ras-induced senescence and tumor suppression. Cell 128: 295-308, 2007.

9. Naylor RM, Baker DJ and van Deursen JM: Senescent cells: A novel therapeutic target for aging and age-related diseases. Clin Pharmacol Ther 93: 105-116, 2013.

10. Coppé JP, Desprez PY, Krtolica A and Campisi J: The senescence-associated secretory phenotype: The dark side of tumor suppression. Annu Rev Pathol 5: 99-118, 2010.

11. Campisi J: Cellular senescence: Putting the paradoxes in perspective. Curr Opin Genet Dev 21: 107-112, 2011.

12. Kang TW, Yevsa T, Woller N, Hoenicke L, Wuestefeld T, Dauch D, Hohmeyer A, Gereke M, Rudalska R, Potapova A, et al: Senescence surveillance of pre-malignant hepatocytes limits liver cancer development. Nature 479: 547-551, 2011.

13. Xue W, Zender L, Miething C, Dickins RA, Hernando E, Krizhanovsky V, Cordon-Cardo C and Lowe SW: Senescence and tumour clearance is triggered by p53 restoration in murine liver carcinomas. Nature 445: 656-660, 2007. 
14. Byun HO, Han NK, Lee HJ, Kim KB, Ko YG, Yoon G, Lee YS, Hong SI and Lee JS: Cathepsin D and eukaryotic translation elongation factor 1 as promising markers of cellular senescence. Cancer Res 69: 4638-4647, 2009.

15. Jung SH, Lee HC, Yu DM, Kim BC, Park SM, Lee YS, Park HJ, Ko YG and Lee JS: Heparan sulfation is essential for the prevention of cellular senescence. Cell Death Differ: Aug 7, 2015 (Epub ahead of print). doi: 10.1038/cdd.2015.107.

16. Kim BC, Yoo HJ, Lee HC, Kang KA, Jung SH, Lee HJ, Lee M, Park S, Ji YH, Lee YS, et al: Evaluation of premature senescence and senescence biomarkers in carcinoma cells and xenograft mice exposed to single or fractionated irradiation. Oncol Rep 31: 2229-2235, 2014.

17. Weichhaus M, Segaran P, Renaud A, Geerts D and Connelly L: Osteoprotegerin expression in triple-negative breast cancer cells promotes metastasis. Cancer Med 3: 1112-1125, 2014.

18. Coussens LM and Werb Z: Inflammation and cancer. Nature 420 $860-867,2002$

19. Vakkila $\mathbf{J}$ and Lotze MT: Inflammation and necrosis promote tumour growth. Nat Rev Immunol 4: 641-648, 2004.

20. Joyce JA: Therapeutic targeting of the tumor microenvironment. Cancer Cell 7: 513-520, 2005.

21. Zender L and Rudolph KL: Keeping your senescent cells under control. Aging (Albany NY) 1: 438-441, 2009.

22. Schmitt CA: Cellular senescence and cancer treatment. Biochim Biophys Acta 1775: 5-20, 2007.

23. de Visser KE, Eichten A and Coussens LM: Paradoxical roles of the immune system during cancer development. Nat Rev Cancer 6: 24-37, 2006.

24. Gewirtz DA, Holt SE and Elmore LW: Accelerated senescence: An emerging role in tumor cell response to chemotherapy and radiation. Biochem Pharmacol 76: 947-957, 2008.

25. Ramkumar C, Kong Y, Trabucco SE, Gerstein RM and Zhang H: Smurf2 regulates hematopoietic stem cell self-renewal and aging. Aging Cell 13: 478-486, 2014.

26. Nardella C, Clohessy JG, Alimonti A and Pandolfi PP. Pro-senescence therapy for cancer treatment. Nat Rev Cancer 11: 503-511, 2011.

27. Velarde MC, Demaria M and Campisi J: Senescent cells and their secretory phenotype as targets for cancer therapy. Interdiscip Top Gerontol 38: 17-27, 2013.

28. Rodier F, Coppé JP, Patil CK, Hoeijmakers WA, Muñoz DP, Raza SR, Freund A, Campeau E, Davalos AR and Campisi J: Persistent DNA damage signalling triggers senescence-associated inflammatory cytokine secretion. Nat Cell Biol 11: 973-979, 2009.

29. Ren JL, Pan JS, Lu YP, Sun P and Han J: Inflammatory signaling and cellular senescence. Cell Signal 21: 378-383, 2009.

30. Novakova Z, Hubackova S, Kosar M, Janderova-Rossmeislova L Dobrovolna J, Vasicova P, Vancurova M, Horejsi Z, Hozak P, Bartek J, et al: Cytokine expression and signaling in drug-induced cellular senescence. Oncogene 29: 273-284, 2010.

31. Orjalo AV, Bhaumik D, Gengler BK, Scott GK and Campisi J: Cell surface-bound IL-1alpha is an upstream regulator of the senescence-associated IL-6/IL-8 cytokine network. Proc Natl Acad Sci USA 106: 17031-17036, 2009.

32. Cichowski K and Hahn WC: Unexpected pieces to the senescence puzzle. Cell 133: 958-961, 2008.

33. Jiang H, Schiffer E, Song Z, Wang J, Zürbig P, Thedieck K, Moes S, Bantel H, Saal N, Jantos J, et al: Proteins induced by telomere dysfunction and DNA damage represent biomarkers of human aging and disease. Proc Natl Acad Sci USA 105: 11299-11304, 2008.

34. Coppé JP, Patil CK, Rodier F, Sun Y, Muñoz DP, Goldstein J, Nelson PS, Desprez PY and Campisi J: Senescence-associated secretory phenotypes reveal cell-nonautonomous functions of oncogenic RAS and the p53 tumor suppressor. PLoS Biol 6: 2853-2868, 2008.
35. Nickoloff BJ, Lingen MW, Chang BD, Shen M, Swift M, Curry J, Bacon P, Bodner B and Roninson IB: Tumor suppressor maspin is up-regulated during keratinocyte senescence, exerting a paracrine antiangiogenic activity. Cancer Res 64: 2956-2961, 2004.

36. Vávrová $\mathbf{J}$ and Rezáčová $\mathbf{M}$ : The importance of senescence in ionizing radiation-induced tumour suppression. Folia Biol (Praha) 57: 41-46, 2011.

37. Sabin RJ and Anderson RM: Cellular Senescence - its role in cancer and the response to ionizing radiation. Genome Integr 2: 7,2011.

38. Han NK, Kim BC, Lee HC, Lee YJ, Park MJ, Chi SG, Ko YG and Lee JS: Secretome analysis of ionizing radiation-induced senescent cancer cells reveals that secreted RKIP plays a critical role in neighboring cell migration. Proteomics 12: 2822-2832, 2012.

39. Baud'huin M, Duplomb L, Teletchea S, Lamoureux F, Ruiz-Velasco C, Maillasson M, Redini F, Heymann MF and Heymann D: Osteoprotegerin: Multiple partners for multiple functions. Cytokine Growth Factor Rev 24: 401-409, 2013.

40. Walsh MC and Choi Y: Biology of the RANKL-RANK-OPG system in immunity, bone, and beyond. Front Immunol 5: 511, 2014.

41. Dougall WC: Molecular pathways: Osteoclast-dependent and osteoclast-independent roles of the RANKL/RANK/OPG pathway in tumorigenesis and metastasis. Clin Cancer Res 18: 326-335, 2012.

42. Reid PE, Brown NJ and Holen I: Breast cancer cells stimulate osteoprotegerin (OPG) production by endothelial cells through direct cell contact. Mol Cancer 8: 49, 2009.

43. Lamoureux F, Moriceau G, Picarda G, Rousseau J, Trichet V and Rédini F: Regulation of osteoprotegerin pro- or anti-tumoral activity by bone tumor microenvironment. Biochim Biophys Acta 1805: 17-24, 2010

44. Kishida S, Mu P, Miyakawa S, Fujiwara M, Abe T, Sakamoto K, Onishi A, Nakamura Y and Kadomatsu K: Midkine promotes neuroblastoma through Notch2 signaling. Cancer Res 73: 1318-1327, 2013

45. Kishida $\mathrm{S}$ and Kadomatsu K: Involvement of midkine in neuroblastoma tumourigenesis. Br J Pharmacol 171: 896-904, 2014.

46. Yao J, Li WY, Li SG, Feng XS and Gao SG: Midkine promotes perineural invasion in human pancreatic cancer. World $\mathrm{J}$ Gastroenterol 20: 3018-3024, 2014.

47. Hao H, Maeda Y, Fukazawa T, Yamatsuji T, Takaoka M, Bao XH, Matsuoka J, Okui T, Shimo T, Takigawa N, et al: Inhibition of the growth factor MDK/midkine by a novel small molecule compound to treat non-small cell lung cancer. PLoS One 8: e71093, 2013.

48. Chen YC, Pohl G, Wang TL, Morin PJ, Risberg B, Kristensen GB, $\mathrm{Yu}$ A, Davidson B and Shih IeM: Apolipoprotein E is required for cell proliferation and survival in ovarian cancer. Cancer Res 65: 331-337, 2005 .

49. Li Y, Cam J and Bu G: Low-density lipoprotein receptor family: Endocytosis and signal transduction. Mol Neurobiol 23: 53-67, 2001.

50. Lynch JR, Morgan D, Mance J, Matthew WD and Laskowitz DT: Apolipoprotein E modulates glial activation and the endogenous central nervous system inflammatory response. J Neuroimmunol 114: 107-113, 2001.

51. Su WP, Chen YT, Lai WW, Lin CC, Yan JJ and Su WC: Apolipoprotein E expression promotes lung adenocarcinoma proliferation and migration and as a potential survival marker in lung cancer. Lung Cancer 71: 28-33, 2011.

52. Wolf AB, Valla J, Bu G, Kim J, LaDu MJ, Reiman EM and Caselli RJ: Apolipoprotein E as a $\beta$-amyloid-independent factor in Alzheimer's disease. Alzheimers Res Ther 5: 38, 2013. 